\title{
Two-year-old children preferentially transmit simple actions but not pedagogically demonstrated actions
}

\author{
Marina Bazhydai $^{1}$ (D) | Priya Silverstein ${ }^{1,2}$ (D) | Eugenio Parise ${ }^{1}$ | Gert Westermann ${ }^{1}$
}

${ }^{1}$ Psychology Department, Lancaster University, Lancaster, UK

${ }^{2}$ School of Psychology, University of Surrey, Guildford, UK

\section{Correspondence}

Marina Bazhydai and Priya Silverstein, Psychology Department, Lancaster University, Lancaster, UK.

Email: m.bazhydai@lancaster.ac.uk; p.silverstein@lancaster.ac.uk

Funding information Leverhulme Trust, Grant/Award Number: DS-2014-14; Economic and Social Research Council, Grant/Award Number: ES/ L008955/1

\begin{abstract}
Children are sensitive to both social and non-social aspects of the learning environment. Among social cues, pedagogical communication has been shown to not only play a role in children's learning, but also in their own active transmission of knowledge. Vredenburgh, Kushnir and Casasola, Developmental Science, 2015, 18, 645 showed that 2-year-olds are more likely to demonstrate an action to a naive adult after learning it in a pedagogical than in a non-pedagogical context. This finding was interpreted as evidence that pedagogically transmitted information has a special status as culturally relevant. Here we test the limits of this claim by setting it in contrast with an explanation in which the relevance of information is the outcome of multiple interacting social (e.g., pedagogical demonstration) and non-social properties (e.g., action complexity). To test these competing hypotheses, we varied both pedagogical cues and action complexity in an information transmission paradigm with 2-year-old children. In Experiment 1, children preferentially transmitted simple non-pedagogically demonstrated actions over pedagogically demonstrated more complex actions. In Experiment 2, when both actions were matched for complexity, we found no evidence of preferential transmission of pedagogically demonstrated actions. We discuss possible reasons for the discrepancy between our results and previous literature showing an effect of pedagogical cues on cultural transmission, and conclude that our results are compatible with the view that pedagogical and other cues interact, but incompatible with the theory of a privileged role for pedagogical cues.
\end{abstract}

\section{KEYWORDS}

action complexity, information transmission, pedagogical demonstrations, social learning

\section{1 | INTRODUCTION}

\begin{abstract}
Social transmission of information is achieved through observation, imitation, and explicit teaching. The human capacity to flexibly engage learners in pedagogical contexts is at the core of transmission of complex cumulative human culture (Burdett, Dean, \& Ronfard, 2017; Caldwell, Renner, \& Atkinson, 2017; Kline, 2015). Although socially mediated learning in early childhood has been studied extensively,
\end{abstract}

Marina Bazhydai and Priya Silverstein contributed equally to this paper little is known about child-initiated teaching (Nakao \& Andrews, 2014; Sobel \& Letourneau, 2015; Ziv \& Frye, 2004) and its cognitive mechanisms (Corriveau, Ronfard, \& Cui, 2017; Paulus, Kim, \& Sodian, 2015). Active transmission of information from children to others starts in infancy (Liszkowski, Carpenter, \& Tomasello, 2008), but the majority of studies on child-initiated teaching focus on preschool to primary school age children (Flynn, Turner, \& Giraldeau, 2016; Flynn \& Whiten, 2012; Whiten \& Flynn, 2010), leaving a gap in understanding the developmental trajectory of information transmission. This emerging body of research shows children's sensitivity to what 
and whom they are teaching (Gweon \& Schulz, 2019; Kim, Kalish, Weisman, Johnson, \& Shutts, 2016; Rhodes, Bonawitz, Shafto, Chen, \& Caglar, 2015; Ronfard, Was, \& Harris, 2016).

While research on information transmission in early childhood is limited, as they acquire knowledge, children are sensitive to both non-social and social aspects of the learning environment. Nonsocial factors include, among others, perceptual salience (Pruden, Hirsh-Pasek, Golinkoff, \& Hennon, 2006), novelty (Mather \& Plunkett, 2012), perceived causality (Burdett, McGuigan, Harrison, \& Whiten, 2018; Ronfard et al., 2016), efficiency (Pinkham \& Jaswal, 2011), and complexity or difficulty(Bannard, Rosner, \& Matthews, 2017; Flynn et al., 2016; Kidd, Piantadosi, \& Aslin, 2012). Such salient factors guide integration of knowledge about objects, causal relations, statistical patterns, and updating and rejecting of hypotheses in light of acquired evidence (Bonawitz, van Schijndel, Friel, \& Schulz, 2012; Gopnik et al., 2017; Waismeyer \& Meltzoff, 2017).

Social factors affecting learning include perceived intentions, competence, past accuracy, and social status of the informant or teacher (for reviews see Harris, 2012; Over \& Carpenter, 2012; Tomasello, 1999). Furthermore, pedagogical cues, such as infant-directed speech (Eaves, Feldman, Griffiths, \& Shafto, 2016), mutual gaze and joint attention (Striano, Chen, Cleveland, \& Bradshaw, 2006), and explicit linguistic cues (Butler \& Tomasello, 2016; Gelman, Ware, Manczak, \& Graham, 2013), have been shown to selectively affect children's learning (Sage \& Baldwin, 2011) in a way that is argued to not be able to be explained by heightened attention (but see Gredebäck, Astor, \& Fawcett, 2018; Szufnarowska, Rohlfing, Fawcett, \& Gredebäck, 2014).

Pedagogical cues have been shown to not only play a role in children's learning, but also in children's own active transmission of knowledge. Vredenburgh, Kushnir, and Casasola (2015) showed that 2-year-olds were more likely to demonstrate an action to a naïve experimenter not present during the demonstrations after learning it in a pedagogical than in a non-pedagogical context. In their study, children were taught two possible actions on one toy. One action was shown in an explicitly pedagogical manner (involving infant-directed speech and direct eye contact), whereas the other action was shown in an intentional but non-pedagogical way. Children were equally likely to learn both of these actions immediately following the demonstrations, but preferentially demonstrated the action that had been shown in a pedagogical context.

A variety of social learning theories propose that a human predisposition for learning from others, mediated by pedagogical cues, enables effective cultural transmission, thereby reducing the costs of unconstrained trial-and-error exploration. These theories predict that knowledge is most likely to be effectively transmitted when it was learnt as a cultural convention (Csibra \& Gergely, 2009; Harris, 2012; Tomasello, 1999, 2016; Vygotsky, 1978). For instance, Tomasello (2016) argues for pedagogical instruction being one of the pillars of cultural learning, with evidence accumulating that pedagogical contexts selectively enable children to generalize such information (e.g., Butler \& Markman, 2012; Gelman et al., 2013).

\section{Research highlights}

- We directly contrasted pedagogical demonstration with complexity of novel actions in a knowledge transmission paradigm with 2-year-old children.

- Children preferentially transmitted simple non-pedagogically demonstrated actions over pedagogically demonstrated complex actions.

- We found no evidence of preferential transmission of pedagogically demonstrated actions when both actions were matched for complexity.

While most pedagogical learning theories merely highlight the importance of pedagogical cues and are not specific about the relationship between these cues and other, non-pedagogical cues, the arguably most prominent such theory, Natural Pedagogy (NP, Csibra \& Gergely, 2009, 2011), argues for a qualitative distinction between pedagogical and non-pedagogical cues. According to NP, humans have evolved to be sensitive to a circumscribed set of pedagogical cues (direct eye gaze, child directed speech, contingent reactivity), which directly create the expectation of communicative intention in the learner, circumventing the need to infer such an intention from other behaviors (Csibra, 2010). In this way pedagogical cues are thought to be a potent driver of infants' learning especially of culturally relevant knowledge that would be hard to acquire relying on observation alone, and their presence leads to the infant's effective encoding of such information. Nevertheless, as a privileged status for pedagogical cues in knowledge transmission is common to all described theories we refer to them here collectively as Privileged Pedagogy (PP) views.

An interpretation of pedagogical cues that is in contrast to views ascribing a qualitatively distinct role to them is that, through their salience, they merely enhance attention to the learning situation without assuming infants' understanding of communicative and referential intentions behind them (Heyes, 2016, 2017). In this way, pedagogical cues interact with other situational factors to affect the likelihood of successful learning. In such a Cue Combination (CC) framework, each cue, weighted by its saliency, has an effect on the likelihood of some information being learned or transmitted, and combined weights of the cues favor learning of one piece of information over another. Such an approach resembles, for instance, the emergentist coalition model of word learning (Hirsh-Pasek, Golinkoff, \& Hollich, 2000), which suggests that children attend to, weigh, and integrate a variety (coalition) of developmentally accessible cues (social, perceptual, cognitive, and purely linguistic) in learning new words. A combination of social and non-social cues has been shown to guide infants' attention, leading to effective learning outcomes in different settings (Barry, Graf Estes, \& Rivera, 2015; Smith, Colunga, \& Yoshida, 2010; Wu \& Kirkham, 2010; Wu, Tummeltshammer, Gliga, \& Kirkham, 2014). Extending this account beyond gradual weighted cue combination and presenting the social 
and non-social cue combination as a complex framework (Yurovsky \& Frank, 2017), it has been proposed that domain general cognitive processes such as attention and speed of information processing explain developmental change in infants' use of social cues during word learning. Relatedly, rational constructivist approaches to social learning also argue for an integrative view, namely accounting for children's existing knowledge (Sobel \& Kushnir, 2013) and statistical inference (Xu \& Kushnir, 2013). Together, these approaches argue for embracing the complexity arising from real life learning situations, and for designing experiments which manipulate several competing cues while taking into account the different weightings of these cues.

Critically, while both PP and CC accounts assume a strong role for pedagogical cues in learning and transmission, they differ in how they explain the mechanismsunderlying the effect of pedagogical cues. Whereas, for example, NP assumes that pedagogical cues have an evolved privileged and specific status in learning that is not based on their salience (Csibra \& Gergely, 2006, 2009, 2011; Senju \& Csibra, 2008; Yoon, Johnson, \& Csibra, 2008), others have argued that indeed the role of pedagogical cues is salience-based and have shown that non-pedagogical salient cues may lead to the same effects (Gredebäck et al., 2018; Szufnarowska et al., 2014). Other work found that previous evidence for a privileged status of pedagogical cues may be difficult to replicate (Silverstein, Gliga, Westermann, \& Parise, 2019), and that both independently accumulated statistical evidence and an explicit pedagogical stance that highlights the importance of information affect the likelihood of successful learning (Buchsbaum, Gopnik, Griffiths, \& Shafto, 2011).

Studies that are aimed at specifically evaluating the function of pedagogical cues typically contrast a pedagogical and a non-pedagogical condition with other cues held constant (e.g., Senju \& Csibra, 2008; Vredenburgh et al., 2015), and can therefore be accounted for both within the PP and CC frameworks. In order to disentangle these two accounts, it is necessary to manipulate both pedagogical and non-pedagogical aspects of the learning situation, but few studies have taken this approach. Previous findings from studies pitting pedagogical against other cues have focused on the efficiency of an action for achieving a specific goal, and have been inconclusive: different studies found selective preference for pedagogical cues (Marno \& Csibra, 2015), equal rates of imitation of pedagogically cued and efficient actions (Brugger, Lariviere, Mumme, \& Bushnell, 2007), imitation of inefficient actions only after pedagogical demonstration but not after independent exploration (Pinkham \& Jaswal, 2011), or effects of pedagogical cues in overriding children's propensity to over-imitate inefficient actions (Hoehl, Zettersten, Schleihauf, Grätz, \& Pauen, 2014).

Building on Vredenburgh et al.'s (2015) finding that 2-year-olds are more likely to demonstrate an action to an adult after learning it in a pedagogical rather than non-pedagogical context, in the present study we set to investigate which of two potentially competing cues-action complexity as a non-social cue and pedagogical demonstration as a social cue-selectively affects the likelihood of an action being shown to an ignorant adult. Although Vredenburgh et al. interpreted their results within a PP framework, we here aimed to directly evaluate $P P$ versus $C C$ accounts by pitting an experimenter's pedagogically demonstrated complex action against another's non-pedagogically demonstrated simple action and observing which action was then preferentially transmitted by the child to a third, naïve experimenter. We focused on 2-year-old children to enable direct comparison between our results and those found by Vredenburgh et al.

While largely adopting Vredenburgh et al.'s (2015) experimental paradigm, we made several crucial changes to the procedure and some of the phrasing to provide a more stringent test of the pedagogical manipulation, by stressing the experimenter's direct, explicit teaching intention. Specifically, we changed the verbal prompt used by the experimenter performing the pedagogical demonstration from "Look! Do you see this?" to "Look at this! This is how you do it!", and the prompt used in the non-pedagogical demonstration from "I like this! Nice!" to the accidental discovery prompt: "What's this? Oh, that's how you do it". Both phrases could (and should) lead to social learning, but the difference lies in demonstrators taking the intentional and norm inducing pedagogical as opposed to intentional but non-directive non-pedagogical stances. We ensured that both demonstrators did not interact with children prior to the experiment to control for potential preferences for one over the other, while the third experimenter acting as an ignorant person, on the contrary, was familiar to them and strongly evoked the conventional, normative context by expecting action transmission from children (saying "Can you show me how to play with it?", which is a change from "What does this do? Can you show me?"). Collectively, these modifications were made to provide a more controlled manipulation and a more specific test of the effect of pedagogy above and beyond normativity inferences made by children from observing demonstrations (e.g., Casler, Terziyan, \& Greene, 2009; Schmidt, Butler, Heinz, $\&$ Tomasello, 2016), while maintaining interactive engagement in the aim of ecological validity.

For the non-social cue, we manipulated action complexity, or difficulty of execution. Simple actions are faster to perform, require less effort, and have a higher probability of achieving the desirable outcome, and thereby, if we assume CC, action simplicity may outweigh the enhanced attention to more complex actions afforded by pedagogical cues. This reasoning is also in line with recent findings that older children transmit information to others in accordance with principle of the utility calculus, making rational and efficient decisions based on the complexity of information and the perceived needs of the naive learner (Bridgers, Jara-Ettinger, \& Gweon, 2019; Gweon \& Schulz, 2019). Note that this approach is in contrast to studies manipulating the efficiency cue, where different manners of executing an action result in the same outcome (Marno \& Csibra, 2015; Pinkham \& Jaswal, 2011). We chose to instead manipulate complexity in order to ensure there were qualitatively different "pieces" of information (i.e., actions with different outcomes) to learn and transmit.

In Experiment 1, we directly contrasted PP and CC explanations for children's choice of action transmission by manipulating 
pedagogical cues and action complexity simultaneously. PP would argue that pedagogically transmitted actions should be transmitted preferentially as pedagogical cues hold a privileged status over other cues (Csibra, 2010; Csibra \& Gergely, 2006). In contrast, from a CC perspective, pedagogical cues and action complexity should interact to lead to children's preferred choice of action based on the relative weighting of the cues, so that pedagogical cues can be outweighed by information complexity. Then, in order to clarify the results of Experiment 1, in Experiment 2 we conducted a replication of Vredenburgh et al.'s (2015) manipulation of pedagogical cues while matching action complexity, retaining other methodological changes outlined in Experiment 1. Data, example videos, and supplementary results can be found on the Open Science Framework (OSF; https:// osf.io/e2hvj/).

\section{EXPERIMENT 1}

\section{1 | Method}

\subsection{1 | Participants}

Thirty-one 24-month-old children from a local community in a small city in Northwestern England participated in the experiment (15 females, $M_{\text {age }}=24$ months 2 days, $S D=0.49$, range 23.2825.25 months). Two more children were tested but not included in the final sample due to failure to perform any actions.

\subsection{2 | Stimuli}

Two unfamiliar toys served as stimuli in two trials (see Figure 1a). Each toy had two target functions, each producing a unique attractive sound. The first toy included two different buttons. One of the buttons was visibly located on the surface of the toy and could be pressed with a finger (simple action). The second button was hidden inside the toy but could be pressed with a hammer-like tool slotted into the toy (complex action). The second toy included a spiral feature, which made a sound when squished down (simple action), and a shaker feature that needed to be removed from the object before shaking to produce the sound (complex action). Toys were designed specifically so that the actions were not obvious, with many distracting non-functional elements. All action outcomes produced comparable sounds that differed in the complexity required to elicit them.

\subsection{3 | Procedure}

The experiment was divided into two phases: a demonstration (including a Pedagogical and a non-pedagogical demonstration and a post-demonstration) and a Transmission phase. Experimenter 1 (E1) briefly interacted with the child during a warm up before the demonstration started. The child was seated in a high chair at a rectangular table, with the caregiver sitting slightly behind and to the side of the child (see Figure 1b). The caregiver was instructed not to interfere in any way. Following the warm up phase, E1 told the child that she would now leave the room and that her friends would like to come in with a new toy that she herself had never seen. She then left the room and closed the door. There were two consecutive trials, one for each toy. Each trial included all phases. The entire procedure was filmed using two video cameras.

\section{Demonstration phase}

Experimenters 2 and 3 (E2 and E3), previously unseen by the child, were assigned to either the pedagogical or non-pedagogical demonstration, with order and condition counterbalanced across trials and participants. Upon E1 leavingthe room, E2 and E3 entered, bringing in the first toy. They sat side by side across the table, facing the child. E2 and E3 each demonstrated one action on the toy three times, either pedagogically (always the complex action) or non-pedagogically (always the simple action). Then (post-demonstration) the experimenter who had just performed the action placed the toy in front of the child and asked: "Can you do it?" If the child performed the demonstrated action within $15 \mathrm{~s}$, the phase was finished, the toy returned to the other end of the table, the other experimenter demonstrated the other action and asked the child to perform it. If the child did not act after $15 \mathrm{~s}$, the experimenter asked: "Can you show me?" Throughout the demonstration, the other experimenter gazed downwards. Upon completing the demonstrations, both experimenters left the table and sat in the far corner of the room behind the child.

\section{Pedagogical demonstration}

The experimenter made frequent eye contact with the child throughout the demonstration and spoke in child-directed speech. After taking a look at the toy, the demonstrator looked at the child, establishing joint attention, and said excitedly: "Look at this! This is how you do it!" She then demonstrated the complex function in a slow, deliberate manner, repeating it three times, alternating her gaze between the child and the object.

\section{Non-pedagogical demonstration}

The experimenter maintained attention on the toy, making no eye contact with the child. She looked at the toy and uttered in a self-addressed manner in an adult-directed speech: "What's this? Oh, that's how you do it". The demonstrator performed the simple function in a slow, deliberate manner and repeated it three times.

\section{Transmission phase}

E1 knocked at the door and re-entered the testing room, greeted the child and showed excitement upon noticing the toy on the table, saying: "Wow! Is that your new toy? That's an interesting toy! I've never seen that before! Can you show me how to play with it?" If the child did not perform any actions after $15 \mathrm{~s}$, the experimenter asked: "Can you show me what this toy does?" After $30 \mathrm{~s}$, the experimenter thanked the child and took the toy away, leaving the testing room. 
(a)

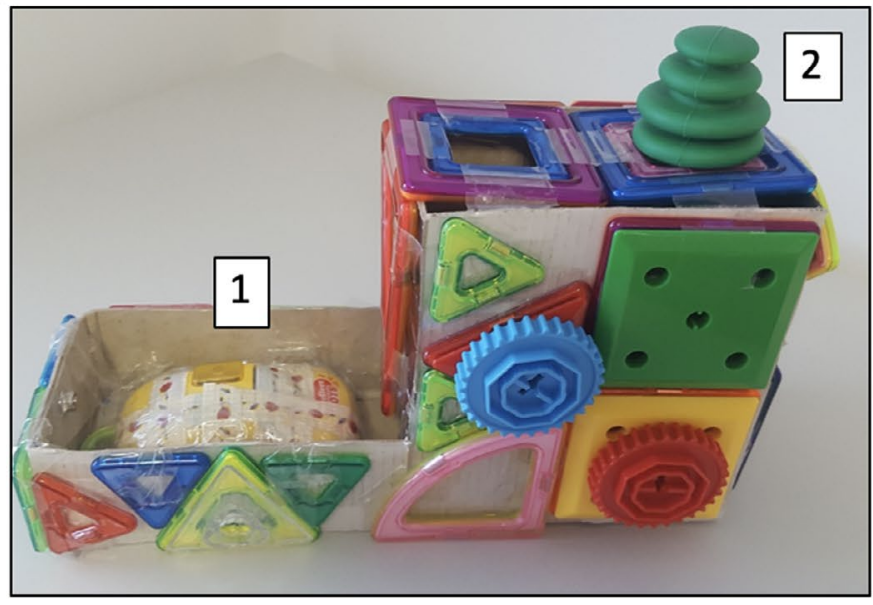

Toy with two simple actions: 1 . Button press on yellow button at the bottom to elicit the sound, 2. Button press on the green button on the top to elicit the sound.

(b)

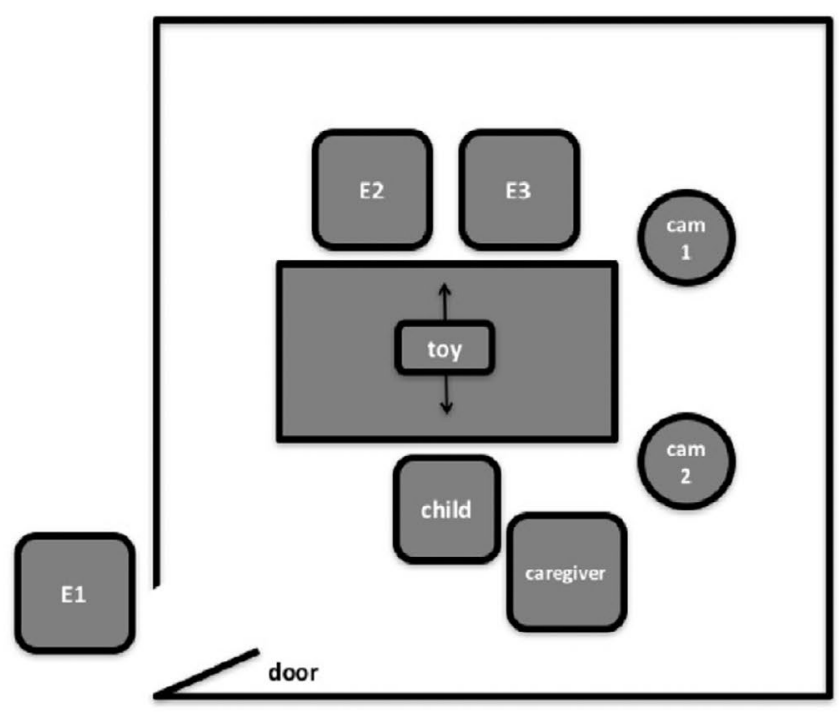

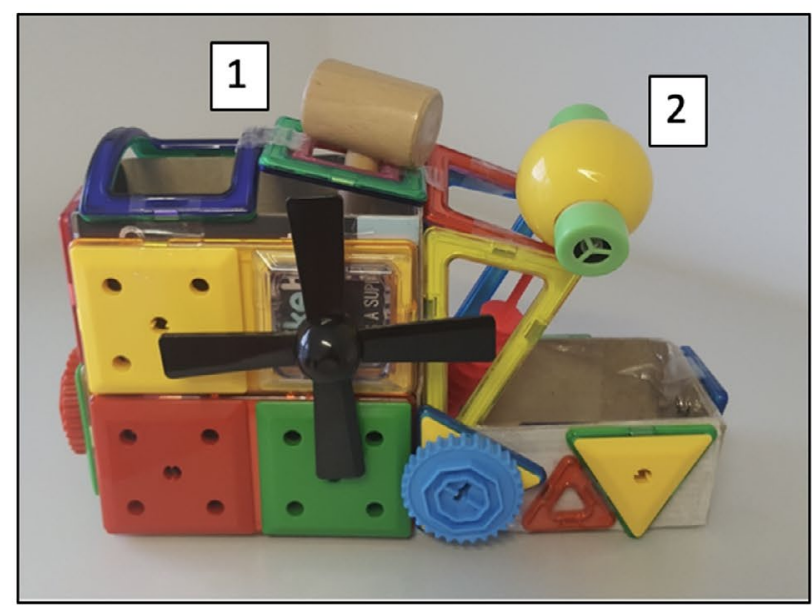

Toy with two complex actions: 1 . Wooden toy is pushed through the opening at the top of the toy to press the button to elicit the sound, 2. Plastic toy is pulled out and shaken to elicit the sound.

(c)

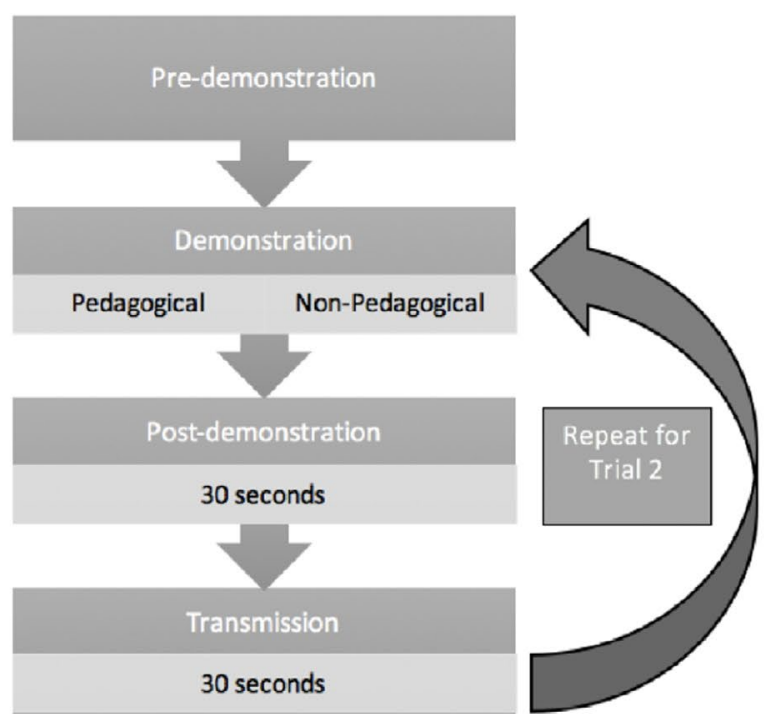

FIGURE 1 Example stimuli (a), schematic of the experimental setup (b), and experimental procedure (c). Example videos can be found on the Open Science Framework

\subsection{4 | Coding}

In order to be included in the final sample, children had to contribute at least one of the two experimental trials. Twenty-five children contributed both trials, and six children contributed one.

\section{Trial exclusion}

Six individual trials were excluded from final analyses due to children's failure to perform any actions $(N=4)$, experimenter error $(N=1)$, and parental interference $(N=1)$.

\section{Post-demonstration}

For each of the four post-demonstrations (two actions by two objects), we coded whether children performed the target action (hereafter called "manipulation check") and whether this resulted in achieving the outcome, that is, sound ("achieving the action outcome"). Note, we have used the terminology "performed" for actions that were attempted by the children regardless of whether they achieved the desired outcome, in contrast to "achieving the action outcome". We created two additional dichotomous variables ("exploratory behaviors"): accidentally discovering the non-target 
action during the first action's post-demonstration, and performing both target actions during the second action's post-demonstration phase.

\section{Transmission phase}

The exact sequence of actions performed in each transmission phase (one per toy/trial) was coded, resulting in two measures: which action was shown first to the experimenter ("first action") and number of switches to each action type ("number of actions").

\section{Inter-rater reliability}

An independent blind coder performed the offline coding. Another researcher double-coded $20 \%$ of the data. We used Cronbach's $\alpha$ (for continuous variables) and Cohen's $\kappa$ (for dichotomous variables) to assess coders' agreement, that were found to be highly reliable, $\alpha=0.89$ and $\kappa=0.84$. All disagreements were resolved in a joint discussion. Where the two coders differed in their coding of continuous variables by over $50 \%$, the video was jointly recoded and a final code produced. When the two coders' responses differed by less than $50 \%$, an average was used.

\section{2 | Results}

Statistical tests used were consistent with those by Vredenburgh et al. (2015).

\subsection{1 | Post-demonstration}

Trial order (and toy) did not have an effect on manipulation check (Pearson's $\chi^{2}=2.8, p=.09$ ) and on achieving the outcome of the action $\left(\chi^{2}=0.85, p=.36\right)$, we therefore combined across these factors for further analyses.

\section{Manipulation check}

Children passed the manipulation check on $86 \%$ of trials (i.e., performed the action after demonstration), and this did not differ between simple non-pedagogically taught actions and pedagogically taught complex actions (McNemar's $p=.69$ ).

\section{Achieving the action outcome}

Children achieved the action outcome on $57 \%$ of trials, and were significantly more likely to achieve the outcome of simple action (this happened in $98 \%$ of the trials) than complex action (only $15 \%$ of trials), Pearson's $\chi^{2}=46.56, p<.001$, despite successfully passing the manipulation check in both types of actions.

\section{Exploratory behaviors}

In $48 \%$ of post-demonstration trials, children accidentally discovered the non-target action, both simple $(N=31)$ and complex $(N=22)$, Pearson's $\chi^{2}=3.9, p=.05$.

\subsection{2 | Transmission}

To test which action the children preferentially selected during this phase we examined two main variables: the choice of the first action and the number of actions.

\section{First action}

First actions from both trials were converted into scores: +1 (only demonstrated complex action first), 0 (one demonstration of each action first), and -1 (only demonstrated simple action first). These scores were compared to chance (0). Children performed the simple non-pedagogically demonstrated action first significantly more than they performed the complex pedagogically demonstrated action first $(t(30)=2.68, p=.01,95 \% \mathrm{Cl}[-0.68,-0.09]$, Cohen's $d=0.49$, paired $t$ test), see Figure $2 a$.

\section{Number of actions}

The majority of children (79\%) performed both actions in both trials. Children performed a higher number of simple $(M=1.61, S D=0.84)$ than complex $(M=1.23, S D=0.5)$ actions, $t(30)=-2.47, p=.02,95 \%$ $\mathrm{Cl}[-0.71,-0.07]$,Cohen's $d=0.55$, paired $t$ test, see Figure 3 .

\section{Exploratory behavior analyses}

The choice of the action transmitted first was not affected by accidental discovery of the non-target action not shown in the demonstration phase (trial 1: Pearson's $\chi^{2}=1.81, p=.18$; trial 2: Pearson's $\chi^{2}=1.54, p=.21$ ), nor by whether the child performed both actions at the transmission phase (trial 1: Pearson's $\chi^{2}=0.05, p=.82$; trial 2: Pearson's $\chi^{2}=1.17, p=.28$ ).

\section{3 | Experiment 1 discussion}

We found support for children's preferential transmission of the simple non-pedagogically demonstrated actions over the pedagogically demonstrated complex actions with two converging measures. Children transmitted the simple non-pedagogically demonstrated action first more often, and they performed more of these actions. Our complexity manipulation was successful, in that children found it difficult to achieve the outcome of the complex actions, which is considered a good manipulation of complexity in other studies of information transmission (e.g., Whiten \& Flynn, 2010). This does, however, make it possible that the actions were so complex that children did not preferentially transmit them due to developmental motor skill deficiencies; it has been previously found that toddlers will not imitate an action that they cannot motorically achieve (Paulus, Hinnius, Vissers, \& Bekkering, 2011, but see Nielsen, 2006). However, in our study, children overwhelmingly transmitted both actions despite preferentially (i.e., first) transmitting the simple action. Likewise, there was no relationship between infants' achieving the action at post-demonstration and later transmitting it: children's preferential transmission of the complex action was not predicted by their successfully achieving 
FIGURE 2 The distribution of first responses to the ignorant adult's request across both transmission trials. From left to right, the responses are ordered by frequency and exclusivity; the number of children whose first response was only the non-pedagogically demonstrated action (both times or once only), to those whose first response was mixed (one of each type), to those whose first response was the only pedagogically demonstrated action (a) Transmission phase: first response

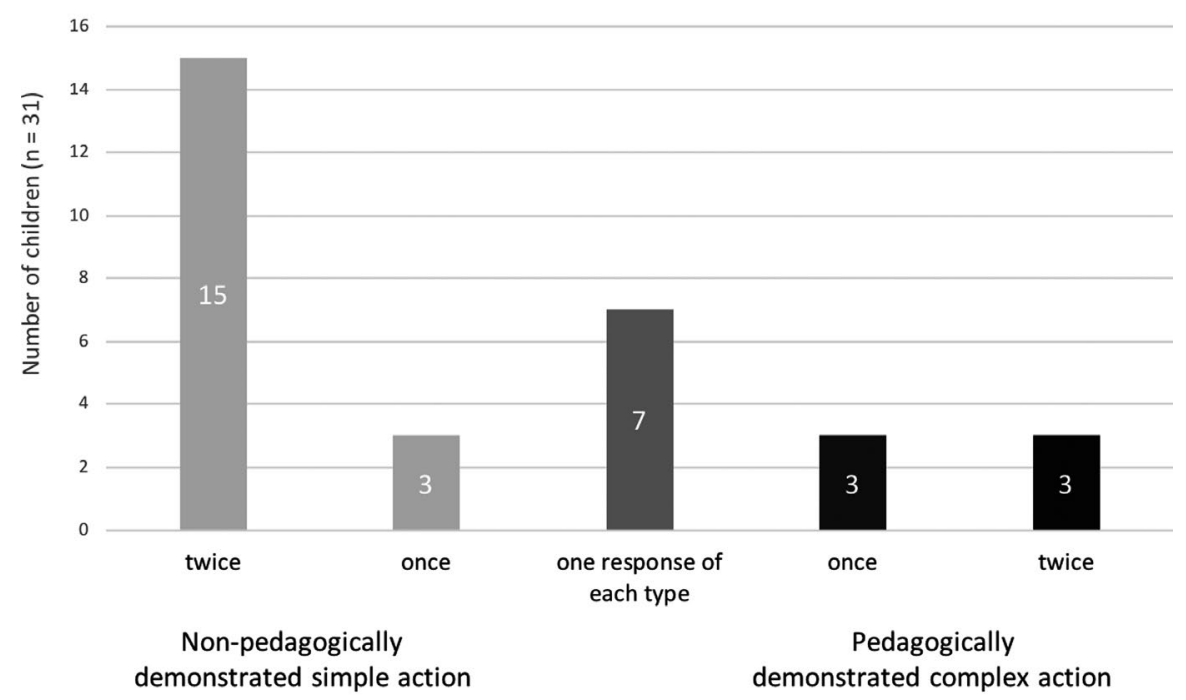

(b)

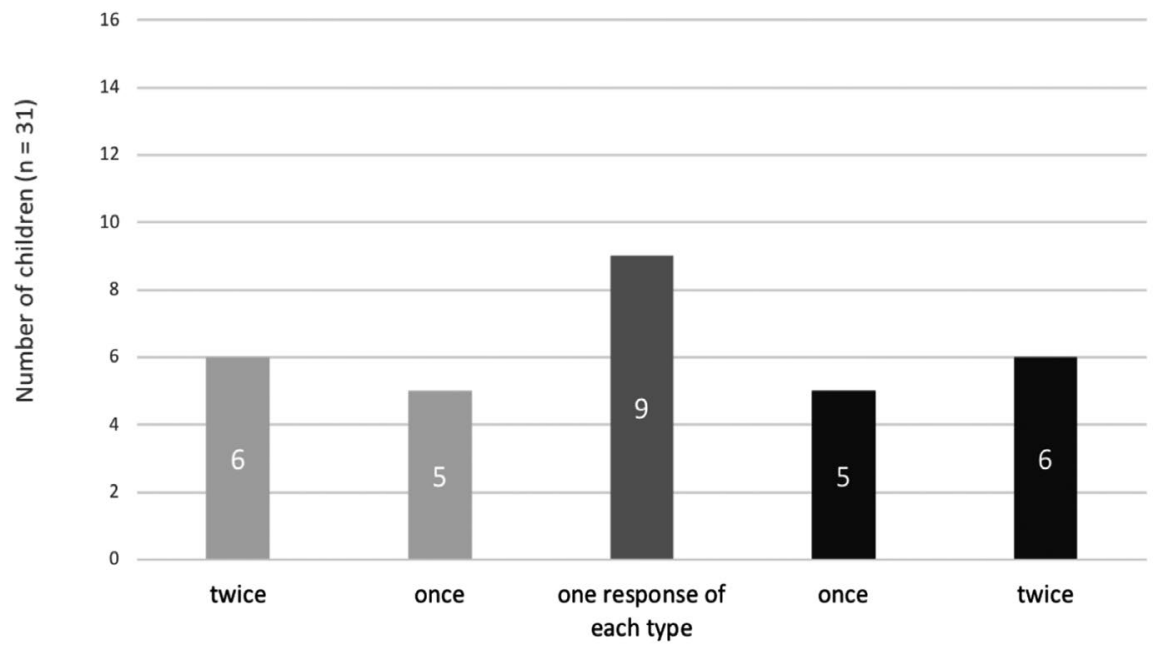

Non-pedagogically demonstrated action

Pedagogically demonstrated action

the outcome: of the 16 children who transmitted the complex action first, four had achieved its outcome at post-demonstration but 12 had not.

These results (in combination with those by Vredenburgh et al., 2015) are best understood within a CC account. When pedagogical cues were manipulated alone, actions demonstrated pedagogically rather than non-pedagogically "won" (Vredenburgh et al., 2015). However, as shown here, when pedagogically demonstrated complex actions were pitted against non-pedagogically demonstrated simple actions, simple actions "won", suggesting that enhanced salience resulting from pedagogical cues is weighted against action simplicity in children's choice of which action to transmit preferentially. This is not to say that it is impossible that even under a CC framework, pedagogical cues could not outweigh simplicity.
However, with our design we could not tell whether our results showed that the properties of the simple action overrode the effect of pedagogical demonstration, or instead whether we, in contrast to Vredenburgh et al. (2015), might find that in our set-up pedagogical teaching does not lead to preferential transmission in the first place. To this end, we decided to conduct a replication of the study by Vredenburgh et al. (2015). We used the same procedure as in Experiment 1, except that action complexity was now matched for both pedagogically and non-pedagogically demonstrated actions in each trial. We thus had one trial with two simple actions (as in Vredenburgh et al., 2015) and the second trial with two complex actions, allowing us to compare how children perform on both pairs at the transmission phase (note that the trial with the simple actions always occurred first in order to ease children into the procedure). 


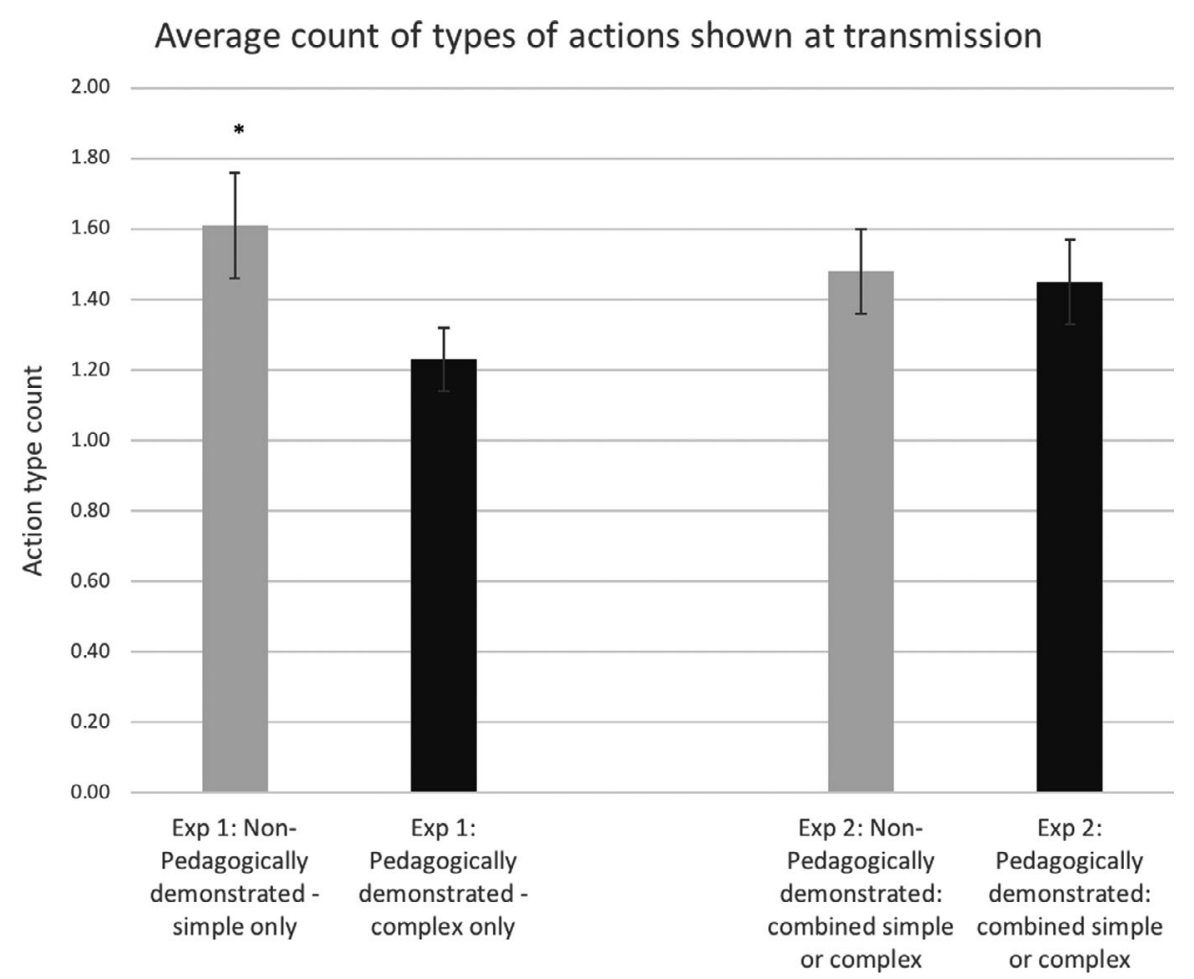

FIGURE 3 Average count of different types of actions shown at transmission phase during both experiments, including pedagogically demonstrated actions and non-pedagogically demonstrated actions. Bars indicate standard errors; ${ }^{*} p<.05$

\section{EXPERIMENT 2}

manipulation was confounded with trial order. Twenty-two children contributed both trials, and nine children contributed one of the trials.

\section{1 | Method}

\subsection{1 | Participants}

Thirty-one 24-month-old children participated in this experiment (14 females, $M_{\text {age }}=24$ months 4 days, $S D=0.36$, range 23.2824.69 months). Three more children were tested but not included in the final sample due to failure to perform any action at post-demonstration or transmission phases $(N=1)$ and parental interference $(N=2)$.

\subsection{2 | Stimuli}

Stimuli were the modified toys from the Experiment 1, where the two functions of each toy were now matched for complexity, with the toy used in the first trial always operated with two simple actions, and the second toy (second trial) operated with a pair of complex actions(see Figure 1a).

\subsection{3 | Procedure and coding}

The procedure was identical to Experiment 1, bar the difference in manipulation (i.e., now actions were matched for complexity rather than contrasted by complexity in each trial). We intentionally did not counterbalance the order of the simple and complex trials so that children would be eased into the procedure with the simple trial. For this reason, we did not compare simple and complex trials statistically, as this

\section{Trial exclusions}

Nine individual trials were excluded from final analyses due to failure to perform any actions $(N=5)$ and experimenter error $(N=4)$.

\section{Coding}

The experimental procedure and coding were identical to Experiment 1 , expect for the addition of action duration as an outcome measure previously shown to be sensitive to this manipulation (Vredenburgh et al., 2015). Thus, the duration of actions performed by the children was manually coded offline frame by frame, from the first frame of the child touching the functional part of the toy. This measure was not reported in Experiment 1 due to the inherent difference in time needed to complete complex and simple actions (these results can be found in Supplementary Materials).

\section{Inter-rater reliability}

The coders achieved high reliability, with continuous variables producing Cronbach's $\alpha=0.87$ and dichotomous variables resulting in Cohen's $\kappa=0.90$.

\section{2 | Results}

\subsection{1 | Post-demonstration}

\section{Manipulation check}

Children passed the manipulation check on $97 \%$ of trials, and the pass rate did not differ between the two actions (McNemar's $p=.69$; 
trial 1/simple: $93 \%$ of children passed the manipulation check; trial 2/complex: $86 \%$ of children).

\section{Achieving the action outcome}

Children were equally able to achieve the outcome of the pedagogically and the non-pedagogically demonstrated actions (McNemar's $p=1$; trial $1 /$ simple: $96 \%$ of children achieved the simple actions outcomes; trial 2/complex: $31 \%$ achieved the complex action outcomes).

\section{Duration}

There were no significant differences in how long children spent executing the target actions in both conditions $(t(51)=0.33, p=.74$, paired $t$ test).

\section{Exploratory behaviors}

In $40 \%$ of post-demonstration trials, children accidentally discovered the non-target action, but this did not differ between pedagogically demonstrated actions and non-pedagogically demonstrated actions in terms of both prevalence (McNemar's $p=.52$ ), and duration of time spent on these actions $(t(50)=0.78, p=.44$, paired $t$ test).

\subsection{2 | Transmission}

\section{First action}

Children did not perform the pedagogically demonstrated action first significantly more than the non-pedagogically demonstrated action $(t(30)=0.00, p=1.0$, paired $t$ test; Figure $2 b)$ or separately for either trial (trial 1/simple: $t(30)=0.00, p=1.0$; trial 2/complex: $t(30)=0.00, p=1.0)$. No statistical inference can be derived from this non-significant result (Lakens, McLatchie, Isager, Scheel, \& Dienes, 2018). Collapsed across trials, a default Bayes factor (Rouder, Morey, Speckman, \& Province, 2012) with a wide Cauchy distribution (scale of effect $=0.707$ ) yielded BFO1 $=3.86$. We can conclude that the data constitute moderate evidence for the null hypothesis (Jeffreys, 1961).

\section{Number of actions}

The majority of children (81\%) performed both actions in both trials. The number of pedagogically and non-pedagogically demonstrated actions did not significantly differ overall $(t(30)=0.40$, $p=.69$, paired $t$ test), or separately for either trial (trial $1 /$ simple: $t(27)=-0.25, p=.80$; trial 2/complex: $t(27)=0.24, p=.81$ ), see Figure 3. Collapsed across trials, a default Bayes factor with a wide Cauchy distribution (scale of effect $=0.707$ ) yielded BF01 $=3.69$, establishing moderate evidence for the null hypothesis. Overall, children performed more actions during trial 1 (simple) than during trial 2 (complex):t(21) $=3.91, p<.001 ; 95 \% \mathrm{Cl}(0.81,2.65)$.

\section{Duration of actions}

Children did not spend significantly longer performing either of the actions overall $(t(30)=0.19, p=.85$, paired $t$ test), or separately for either trial (trial $1 /$ simple: $t(27)=-0.50, p=.62$; trial $2 /$ complex: $t(24)=0.22, p=.83)$. Collapsed across trials, a default Bayes factor with a wide Cauchy distribution (scale of effect $=0.707$ ) yielded moderate support for the null hypothesis, BF01 $=3.79$.

\section{Exploratory behavior analyses}

Whether or not a child accidentally discovered the action that was not shown to them in the demonstration phase (trial 1: Pearson's $\chi^{2}=0.70, p=.40$; trial 2: Pearson's $\chi^{2}=0.71, p=.79$ ), and whether or not a child performed both actions at the transmission phase did not affect which action they chose to transmit first (trial 1: Pearson's $\chi^{2}=0.00, p=1.00 ;$ trial 2: Pearson's $\chi^{2}=0.52, p=.47$ ).

\section{3 | Experiment 2 discussion}

Contrary to the results by Vredenburgh et al. (2015), we found no evidence of preferential transmission of pedagogically demonstrated actions even when both actions were matched for complexity, indexed through first action, duration of actions, or number of actions. This overall result was also found separately for trials with two matched simple actions and two complex actions. This finding is supported by Bayes Factor Analysis, which shows moderate support for the null hypothesis.

Critically, even in the second trial, where both actions were complex, children still transmitted both actions, despite the outcomes only being achieved by $31 \%$ of children at post-demonstration and $40 \%$ of children at transmission phase. Importantly, ignoring all possible effects of our complexity manipulation, even in the simple trial (which is comparable to the actions used in Vredenburgh et al.), we saw no preferential transmission of the pedagogically demonstrated action.

There are several possibilities for why we did not replicate Vredenburgh et al.'s (2015) finding. First, it is possible that our finding is a Type 2 error. However, this is unlikely given Bayes Factor Analysis showing moderate support for the null hypothesis across the range of measures used, and very similar results across the simple and complex action trials. We stress that the changes to the pedagogy manipulation that we made in our design, as compared to Vredenburgh et al., aimed for an even stronger and more stringent comparison of pedagogical versus non-pedagogical demonstration, making us more likely to detect the differences between the manipulations. It is also possible that the original finding was a Type 1 error. As no other studies to date have replicated this finding, this remains a possibility. Future research could investigate the subtleties required for reproducing this effect, and whether the underlying theory should be modified accordingly to include these specific constraints.

Another possibility is that there are key differences between Vredenburgh et al.'s (2015) and our experiment. For example, it is possible that our verbal prompts in effect meant that both conditions were considered normative by the children, in that both prompts contained "this/that is how you do it". In our attempt to create a more controlled manipulation of pedagogical cues, whereby 
the only difference between prompts was the pedagogical nature, we may have inadvertently created a more normative context for both conditions. Nevertheless, normativity is not the defining feature of pedagogical context, and in PP a pedagogical transmission of normative information would still be preferred over non-pedagogical transmission of equally normative information (Csibra, 2010; Csibra \& Gergely, 2009). The defining features of pedagogical context (which we did manipulate) are the presence of ostensive cues and explicit teaching. Future research can disentangle the effect of pedagogical context and normativity in information transmission.

Within the proposed CC framework, it is also possible that differences in the specific pedagogical cues and specific actions used in the two studies alter the specific weighting of cues. In our Experiment 2 , the inherent interest of the actions may have outweighed the salience difference introduced by manipulating pedagogical cues, whereas in the study by Vredenburgh et al., this was not the case. An analogy to illustrate this possibility is a child receiving two presents for their birthday, one accompanied by "pedagogical" cues ("Wow, look at this, a present for you!"), and the other just placed in front of the child. The child might be so excited about the presents per se that the manner (social context) in which they were delivered makes no discernible difference to their interest in them.

Finally, it is possible that, in contrast to Vredenburgh et al.'s (2015) finding, pedagogical cues do not modulate preferential transmission of actions. In light of strong evidence that explicit pedagogical cues are of special importance to children in a variety of learning situations (Butler \& Markman, 2012, 2014, 2016; Csibra \& Gergely, 2009; Marno \& Csibra, 2015) this would be surprising. Nevertheless, Vredenburgh et al's study was the first to extend the notion of the importance of pedagogical cues from learning to a child's active transmission of information, and it is possible that factors that are important in learning do not necessarily translate to information transmission, or do so at a later age than currently investigated. Future research should therefore focus on examining the contexts under which different cues combine to affect transmission.

\section{4 | GENERAL DISCUSSION}

In this study we investigated how two types of cues (pedagogical demonstration and action complexity) affect children's transmission of recently demonstrated actions to a naïve adult, testing the prediction of a Privileged Pedagogy (PP) account that pedagogical cues would ensure preferential encoding of the action even despite its higher complexity, against a Cue Combination (CC) view that both pedagogical and complexity cues would be weighted to determine which action was preferentially transmitted. We report evidence that children preferentially transmitted simple non-pedagogically taught actions over pedagogically taught complex actions (Experiment 1), and no evidence of preferential transmission of pedagogically demonstrated actions when both actions were matched for complexity (Experiment 2), with moderate support for the hypothesis that pedagogical cues have no effect on preferential transmission.
Our results are compatible with the $C C$ view, and incompatible with the PP view. The CC framework can explain this pattern of results, and the difference of our results compared to those of Vredenburgh et al. (2015), if as well as the cues that we purposefully manipulated (action complexity and pedagogical demonstration) we also take into account the intrinsic properties of the toy. It is possible that compared to Vredenburgh et al. (2015), the inherent salience of our toys and our actions was higher. In this way, even if pedagogical demonstration did have some weighting, this did not incur a big enough difference to affect children's preference for action transmission. This is in contrast to Experiment 1, where there are intrinsic action properties that enhance the likelihood of transmission of the simple action. However, we note that although our results are compatible with the $\mathrm{CC}$ view in this way, future research should quantify and examine in more detail the relative weightings of different cues in determining outcomes for learning and transmission.

Several features of our study and that by Vredenburgh and colleagues warrant discussion and further research. First, it is possible that children were not sensitive to the pedagogy manipulation and instead considered the whole demonstration phase as a uniform learning episode, where one demonstrator is ostensibly more communicative than the other. Although we manipulated pedagogy explicitly, using various established cues, a more extreme manipulation of pedagogical and non-pedagogical cues may help resolve this consideration (e.g., Marno \& Csibra, 2015). However, this may be at the expense of ecological validity, and would add further confounds that could be responsible for any differences found (as opposed to the presence of pedagogical cues themselves).

As we chose to manipulate the complexity of the actions by varying their ease and transparency of execution, this resulted in the complex actions being harder to achieve for some of the participants. Although this raises the possibility that it discouraged some children from choosing to transmit complex actions regardless of the manner in which they were demonstrated, three main points speak against this interpretation. In Experiment 1, in trials where children transmitted the complex action preferentially (16), they were no more likely to have been able to achieve the outcome at post-demonstration than not ( 4 achieved, 12 did not). In Experiment 2, the results for the trial in which both actions were complex showed that even with low achievability, infants still transmitted both actions. In the trial where both actions were simple (comparable to Vredenburgh et al., 2015), children showed the same pattern: there was no difference in their transmission of pedagogically and non-pedagogically demonstrated actions. Hence, there is no evidence that being unable to achieve the outcome of the complex action affected children's transmission choice. Future research should investigate the relationship between the ability to achieve the outcome of an action and its transmission, with a large enough sample to statistically examine these individual differences.

Another promising avenue for future research is cultural transmission of different types of information (Corriveau, Ronfard, et al., 2017; Flynn \& Smith, 2012; Paulus et al., 2015; Ronfard \& Harris, 2018). In the present study, we investigated easy to acquire, 
developmentally appropriate, rewarding, causally unambiguous, accurate, and easily verifiable information. It is an open question whether the mechanisms for transmission of this type of information would be the same as for information that is causally opaque, inefficient or irrelevant (e.g., Burdett et al., 2018; Corriveau, DiYanni, et al., 2017; Lyons, Damrosch, Lin, Macris, \& Keil, 2011; Ronfard et al., 2016), socially conforming and more frequently endorsed (e.g., Morgan, Laland, \& Harris, 2015), or tabooed (e.g., Seehagen, Schneider, Miebach, Frigge, \& Zmyj, 2017). It would also be important to further investigate what is meant by transmission in this paradigm,that is, whether the children are demonstrating what they can do, or that they know the rules, to show what they themselves have learnt, or to explicitly teach someone else. These are among the exciting questions open for investigation.

Like Vredenburgh et al. (2015), we focused on 2-year-olds as the youngest group previously shown to engage in active cultural information transmission, yet a rarely studied population compared to a large body of research with preschool and elementary school children. We still know very little about the developmental trajectory of cultural information transmission in children as they navigate the social world, as well as the extent of the claims made by the PP accounts in toddlers, rather than infants or preschoolers, which should be addressed in further research. As we set out to investigate children's selectivity in social learning, we would like to bear in mind that within a complex, dynamic, self-organizing developmental system (Smith \& Thelen, 2003), mechanisms are constantly changing. It is plausible that while we showed the parsimonious outcome of the present study's manipulation with 2-year-olds, later in development this may change. Understanding when and why such a change occurs is an avenue open for further research.

Overall, our study provided an ecologically valid manipulation of pedagogical cues and evaluated two competing accounts of their role in the learning and transmission of information in toddlers. In our paradigm, children were not influenced by the context of the demonstration, but instead by the properties of the actions themselves, favoring actions that are easy to perform, require less effort, have a higher probability of achieving the desirable outcome, and result in a rewarding sound most readily. What we can conclude is that we do not have support for $P P$, as this view would assume that pedagogical cues should not only have a positive effect on transmission when manipulated alone, but that they should also override other cues, neither of which we were able to show in our two experiments. This study contributes to our understanding of the factors affecting information transmission in early childhood.

\section{ACKNOWLEDGEMENTS}

We thank all participating families and the Lancaster Babylab, as well as research assistants: Charlotte Smallman, Charlotte Rothwell, Kate Milan-Snee, and Hannah Thomas. This work was supported by the Leverhulme Trust Doctoral Scholarship Programme DS-2014-14 and the ESRC International Centre for Language and Communicative Development (LuCiD), [ES/L008955/1]. We thank Tamar Kushnir and an anonymous reviewer for their helpful comments on this paper.

\section{CONFLICT OF INTEREST}

Authors declare no conflict of interest.

\section{AUTHOR CONTRIBUTIONS}

$\mathrm{MB}, \mathrm{PS}, \mathrm{GW}$, and EP designed the study and wrote the manuscript. $M B$ recruited and booked children for the experiment. $M B$ and PS carried out the experiments. MB handled the data and coded the videos. MB performed all Frequentist analyses, and PS performed all Bayesian analyses.

\section{DATA AVAILABILITY STATEMENT}

The data that support the findings of this study are openly available in the Open Science Framework at https://osf.io/e2hvj/.

\section{ORCID}

Marina Bazhydai iD https://orcid.org/0000-0001-9619-3975

Priya Silverstein (iD https://orcid.org/0000-0003-0095-339X

\section{REFERENCES}

Bannard, C., Rosner, M., \& Matthews, D. (2017). What's worth talking about? Information theory reveals how children balance informativeness and ease of production. Psychological Science, 28(7), 954-966. https://doi.org/10.1177/0956797617699848

Barry, R. A., Graf Estes, K., \& Rivera, S. M. (2015). Domain general learning: Infants use social and non-social cues when learning object statistics. Frontiers in Psychology, 6, 1-10. https://doi.org/10.3389/ fpsyg.2015.00551

Bonawitz, E. B., van Schijndel, T. J., Friel, D., \& Schulz, L. (2012). Children balance theories and evidence in exploration, explanation, and learning. Cognitive Psychology, 64(4), 215-234. https://doi.org/10.1016/j. cogpsych.2011.12.002

Bridgers, S., Jara-Ettinger, J., \& Gweon, H. (2019). Young children consider the expected utility of others' learning to decide what to teach. Nature Human Behaviour, 1-9, https://doi.org/10.1038/ s41562-019-0748-6

Brugger, A., Lariviere, L. A., Mumme, D. L., \& Bushnell, E. W. (2007). Doing the right thing: Infants' selection of actions to imitate from observed event sequences. Child Development, 78(3), 806-824. https ://doi.org/10.1111/j.1467-8624.2007.01034.x

Buchsbaum, D., Gopnik, A., Griffiths, T. L., \& Shafto, P. (2011). Children's imitation of causal action sequences is influenced by statistical and pedagogical evidence. Cognition, 120(3), 331-340. https://doi. org/10.1016/j.cognition.2010.12.001

Burdett, E. R., Dean, L. G., \& Ronfard, S. (2017). A diverse and flexible teaching toolkit facilitates the human capacity for cumulative culture. Review of Philosophy and Psychology, 9(4), 807-818. https://doi. org/10.1007/s13164-017-0345-4

Burdett, E. R., McGuigan, N., Harrison, R., \& Whiten, A. (2018). The interaction of social and perceivable causal factors in shaping 'over-imitation'. Cognitive Development, 47, 8-18. https://doi.org/10.1016/j. cogdev.2018.02.001

Butler, L. P., \& Markman, E. M. (2012). Preschoolers use intentional and pedagogical cues to guide inductive inferences and exploration. Child Development, 83, 1416-1428. https://doi. org/10.1111/j.1467-8624.2012.01775.x

Butler, L. P., \& Markman, E. M. (2014). Preschoolers use pedagogical cues to guide radical reorganization of category knowledge. Cognition, 130, 116-127. https://doi.org/10.1016/j.cognition.2013.10.002

Butler, L. P., \& Markman, E. M. (2016). Navigating pedagogy: Children's developing capacities for learning from pedagogical interactions. 
Cognitive Development, 38, 27-35. https://doi.org/10.1016/j. cogdev.2016.01.001

Butler, L. P., \& Tomasello, M. (2016). Two- and 3-year-old children integrate linguistic and pedagogical cues in guiding inductive generalizations and exploration. Journal of Experimental Child Psychology, 145, 64-78. https://doi.org/10.1016/j.jecp.2015.12.001

Caldwell, C. A., Renner, E., \& Atkinson, M. (2017). Human teaching and cumulative cultural evolution. Review of Philosophy and Psychology, 9(4), 751-770. https://doi.org/10.1007/s13164-017-0346-3

Casler, K., Terziyan, T., \& Greene, K. (2009). Toddlers view artifact function normatively. Cognitive Development, 24, 240-247. https://doi. org/10.1016/j.cogdev.2009.03.005

Corriveau, K. H., DiYanni, C. J., Clegg, J. M., Min, G., Chin, J., \& Nasrini, J. (2017). Cultural differences in the imitation and transmission of inefficient actions. Journal of Experimental Child Psychology, 161, 1-18. https://doi.org/10.1016/j.jecp.2017.03.002

Corriveau, K. H., Ronfard, S., \& Cui, Y. K. (2017). Cognitive mechanisms associated with children's selective teaching. Review of Philosophy and Psychology, 9(4), 831-848. https://doi.org/10.1007/s13164-017-0343-6

Csibra, G. (2010). Recognizing communicative intentions in infancy. Mind \& Language, 25(2), 141-168. https://doi.org/10.1111/j.1468-0017. 2009.01384.x

Csibra, G., \& Gergely, G. (2006). Social learning and social cognition: The case for pedagogy. In Y. Munakata \& M. H. Johnson (Eds.), Processes of change in brain and cognitive development. Attention and performance (Vol. XXI, pp. 249-274). Oxford, UK: Oxford University Press.

Csibra, G., \& Gergely, G. (2009). Natural pedagogy. Trends in Cognitive Sciences, 13, 148-153. https://doi.org/10.1016/j.tics.2009.01.005

Csibra, G., \& Gergely, G. (2011). Natural pedagogy as evolutionary adaptation. Philosophical Transactions of the Royal Society B: Biological Sciences, 366, 1149-1157. https://doi.org/10.1098/rstb.2010.0319

Eaves, B. S. Jr., Feldman, N. H., Griffiths, T. L., \& Shafto, P. (2016). Infantdirected speech is consistent with teaching. Psychological Review, 123(6), 758. https://doi.org/10.1037/rev0000031

Flynn, E., Turner, C., \& Giraldeau, L. A. (2016). Selectivity in social and asocial learning: Investigating the prevalence, effect and development of young children's learning preferences. Philosophical Transactions of the Royal Society B: Biological Sciences, 371(1690), 20150189. https:// doi.org/10.1098/rstb.2015.0189

Flynn, E., \& Smith, K. (2012). Investigating the mechanisms of cultural acquisition. Social Psychology, 43, 185-195. https://doi. org/10.1027/1864-9335/a000119

Flynn, E., \& Whiten, A. (2012). Experimental "microcultures" in young children: Identifying biographic, cognitive, and social predictors of information transmission. Child Development, 83(3), 911-925. https ://doi.org/10.1111/j.1467-8624.2012.01747.x

Gelman, S. A., Ware, E. A., Manczak, E. M., \& Graham, S. A. (2013). Children's sensitivity to the knowledge expressed in pedagogical and nonpedagogical contexts. Developmental Psychology, 49(3), 491-504. https://doi.org/10.1037/a0027901

Gopnik, A., O'Grady, S., Lucas, C. G., Griffiths, T. L., Wente, A., Bridgers, S., ... Dahl, R. E. (2017). Changes in cognitive flexibility and hypothesis search across human life history from childhood to adolescence to adulthood. Proceedings of the National Academy of Sciences of the United States of America, 114(30), 7892-7899. https://doi. org/10.1073/pnas.1700811114

Gredebäck, G., Astor, K., \& Fawcett, C. (2018). Gaze following is not dependent on ostensive cues: A critical test of natural pedagogy. Child Development, 89(6), 2091-2098. https://doi.org/10.1111/cdev.13026

Gweon, H., \& Schulz, L. E. (2019). From exploration to instruction: Children learn from exploration and tailor their demonstrations to observers' goals and competence. Child Development, 90(1), 148164. https://doi.org/10.1111/cdev.13059

Harris, P. L. (2012). Trusting what you're told. Cambridge: Belknap/ Harvard.
Heyes, C. (2016). Born pupils? Natural pedagogy and cultural pedagogy. Perspectives on Psychological Science, 11(2), 280-295. https://doi. org/10.1177/1745691615621276

Heyes, C. (2017). When does social learning become cultural learning? Developmental Science, 20(2), e12350. https://doi.org/10.1111/ desc.12350

Hirsh-Pasek, K., Golinkoff, R. M., \& Hollich, G. (2000). An emergentist coalition model for word learning. In R. M. Golinkoff, K. Hirsh-Pasek, L. Bloom, L. B. Smith, A. L. Woodward, N. Akhtar, M. Tomasello, \& G. Hollich (Eds.), Becoming a word learner: A debate on lexical acquisition (pp. 136-164). Oxford, UK: Oxford University Press.

Hoehl, S., Zettersten, M., Schleihauf, H., Grätz, S., \& Pauen, S. (2014). The role of social interaction and pedagogical cues for eliciting and reducing overimitation in preschoolers. Journal of Experimental Child Psychology, 122, 122-133. https://doi.org/10.1016/j.jecp.2013.12.012

Jeffreys, H. (1961). Theory of probability (3rd edn.). Oxford, UK: Oxford University Press.

Kidd, C., Piantadosi, S. T., \& Aslin, R. N. (2012). The Goldilocks effect: Human infants allocate attention to visual sequences that are neither too simple nor too complex. PLoS ONE, 7(5), e36399. https://doi. org/10.1371/journal.pone.0036399

Kim, S., Kalish, C. W., Weisman, K., Johnson, M. V., \& Shutts, K. (2016). Young children choose to inform previously knowledgeable others. Journal of Cognition and Development, 17(2), 320-340. https://doi. org/10.1080/15248372.2014.952731

Kline, M. A. (2015). How to learn about teaching: An evolutionary framework for the study of teaching behavior in humans and other animals. Behavioral and Brain Sciences, 38, https://doi.org/10.1017/S0140 $525 \times 14000090$

Lakens, D., McLatchie, N., Isager, P. M., Scheel, A. M., \& Dienes, Z. (2018). Improving inferences about null effects with bayes factors and equivalence tests. The Journals of Gerontology: Series B, 75(1), 45-57. https://doi.org/10.1093/geronb/gby065

Liszkowski, U., Carpenter, M., \& Tomasello, M. (2008). Twelve-montholds communicate helpfully and appropriately for knowledgeable and ignorant partners. Cognition, 108, 732-739. https://doi. org/10.1016/j.cognition.2008.06.013

Lyons, D. E., Damrosch, D. H., Lin, J. K., Macris, D. M., \& Keil, F. C. (2011). The scope and limits of overimitation in the transmission of artefact culture. Philosophical Transactions of the Royal Society B: Biological Sciences, 366(1567), 1158-1167. https://doi.org/10.1098/rstb.2010.0335

Marno, H., \& Csibra, G. (2015). Toddlers favor communicatively presented information over statistical reliability in learning about artifacts. PLoS ONE, 10(3), e0122129. https://doi.org/10.1371/journ al.pone.0122129

Mather, E., \& Plunkett, K. (2012). The role of novelty in early word learning. Cognitive Science, 36(7), 1157-1177. https://doi.org/10. 1111/ j.1551-6709.2012.01239.x

Morgan, T. J., Laland, K. N., \& Harris, P. L. (2015). The development of adaptive conformity in young children: Effects of uncertainty and consensus. Developmental Science, 18(4), 511-524. https://doi.org/10.1111/ desc.12231

Nakao, H., \& Andrews, K. (2014). Ready to teach or ready to learn: Acritique of the natural pedagogy theory. Review of Philosophy and Psychology, 5(4), 465-483. https://doi.org/10.1007/s13164-014-0187-2

Nielsen, M. (2006). Copying actions and copying outcomes: Social learning through the second year. Developmental Psychology, 42(3), 555565. https://doi.org/10.1037/0012-1649.42.3.555

Over, H., \& Carpenter, M. (2012). Putting the social into social learning: Explaining both selectivity and fidelity in children's copying behavior. Journal of Comparative Psychology, 126(2), 182-192. https://doi. org/10.1037/a0024555

Paulus, M., Hunnius, S., Vissers, M., \& Bekkering, H. (2011). Imitation in infancy: Rational or motor resonance? Child Development, 82(4), 1047-1057. https://doi.org/10.1111/j.1467-8624.2011.01610.x 
Paulus, M., Kim, S., \& Sodian, B. (2015). Clarifying the range of social-cognitive processes subserving human teaching. Behavioral and Brain Sciences, 38, https://doi.org/10.1017/S0140525X14000569

Pinkham, A. M., \& Jaswal, V. K. (2011). Watch and learn? Infants privilege efficiency over pedagogy during imitative learning. Infancy, 16(5), 535-544. https://doi.org/10.1111/j.1532-7078.2010.00059.x

Pruden, S. M., Hirsh-Pasek, K., Golinkoff, R. M., \& Hennon, E. A. (2006). The birth of words: Ten-month-olds learn words through perceptual salience. Child Development, 77(2), 266-280. https://doi. org/10.1111/j.1467-8624.2006.00869.x

Rhodes, M., Bonawitz, E., Shafto, P., Chen, A., \& Caglar, L. (2015). Controlling the message: Preschoolers' use of information to teach and deceive others. Frontiers in Psychology, 6, 867-873. https://doi. org/10.3389/fpsyg.2015.00867

Ronfard, S., \& Harris, P. L. (2018). Children's decision to transmit information is guided by their evaluation of the nature of that information. Review of Philosophy and Psychology, 9(4), 849-861. https://doi. org/10.1007/s13164-017-0344-5

Ronfard, S., Was, A. M., \& Harris, P. L. (2016). Children teach methods they could not discover for themselves. Journal of Experimental Child Psychology, 142, 107-117. https://doi.org/10.1016/j. jecp.2015.09.032

Rouder, J., Morey, R., Speckman, P., \& Province, J. (2012). Default Bayes factors for ANOVA designs. Journal of Mathematical Psychology, 56(5), 356-374. https://doi.org/10.1016/j.jmp.2012.08.001

Sage, K. D., \& Baldwin, D. (2011). Disentangling the social and the pedagogical in infants' learning about tool-use. Social Development, 20(4), 825-844. https://doi.org/10.1111/j.1467-9507.2011.00624.x

Schmidt, M. F., Butler, L. P., Heinz, J., \& Tomasello, M. (2016). Young children see a single action and infer a social norm: Promiscuous normativity in 3-year-olds. Psychological Science, 27(10), 1360-1370. https ://doi.org/10.1177/0956797616661182

Seehagen, S., Schneider, S., Miebach, K., Frigge, K., \& Zmyj, N. (2017). "Should I or shouldn't I?" Imitation of undesired versus allowed actions from peer and adult models by 18 -and 24-month-old toddlers. Infant Behavior and Development, 49, 1-8. https://doi.org/10.1016/j. infbeh.2017.06.001

Senju, A., \& Csibra, G. (2008). Gaze following in human infants depends on communicative signals. Current Biology, 18(9), 668-671. https:// doi.org/10.1016/j.cub.2008.03.059

Silverstein, P., Gliga, T., Westermann, G., \& Parise, E. (2019). Probing communication-induced memory biases in preverbal infants: Two replication attempts of Yoon, Johnson and Csibra (2008). Infant Behavior and Development, 55, 77-87. https://doi.org/10.1016/j. infbeh.2019.03.005

Smith, L. B., Colunga, E., \& Yoshida, H. (2010). Knowledge as process: Contextually cued attention and early word learning. Cognitive Science, 34(7), 1287-1314. https://doi. org/10.1111/j.1551-6709.2010.01130.x

Smith, L. B., \& Thelen, E. (2003). Development as a dynamic system. Trends in Cognitive Sciences, 7(8), 343-348. https://doi.org/10.1016/ S1364-6613(03)00156-6

Sobel, D. M., \& Kushnir, T. (2013). Knowledge matters: How children evaluate the reliability of testimony as a process of rational inference. Psychological Review, 120(4), 779-797. https://doi.org/10.1037/ a0034191

Sobel, D. M., \& Letourneau, S. M. (2015). Children's developing understanding of what and how they learn. Journal of Experimental Child Psychology, 132, 221-229. https://doi.org/10.1016/j. jecp.2015.01.004
Striano, T., Chen, X., Cleveland, A., \& Bradshaw, S. (2006). Joint attention social cues influence infant learning. European Journal of Developmental Psychology, 3(3), 289-299. https://doi.org/10.1080/1740562060 0879779

Szufnarowska, J., Rohlfing, K. J., Fawcett, C., \& Gredebäck, G. (2014). Is ostension any more than attention? Scientific Reports, 4, 5304. https ://doi.org/10.1038/srep05304

Tomasello, M. (1999). The cultural origins of human cognition. Cambridge, MA: Harvard University Press.

Tomasello, M. (2016). The ontogeny of cultural learning. Current Opinion in Psychology, 8, 1-4. https://doi.org/10.1016/j.copsyc.2015.09.008

Vredenburgh, C., Kushnir, T., \& Casasola, M. (2015). Pedagogical cues encourage toddlers' transmission of recently demonstrated functions to unfamiliar adults. Developmental Science, 18(4), 645-654. https:// doi.org/10.1111/desc.12233

Vygotsky, L. S. (1978). Mind in society: The development of higher mental process. Cambridge, MA: Harvard University.

Waismeyer, A., \& Meltzoff, A. N. (2017). Learning to make things happen: Infants' observational learning of social and physical causal events. Journal of Experimental Child Psychology, 162, 58-71. https://doi. org/10.1016/j.jecp.2017.04.018

Whiten, A., \& Flynn, E. (2010). The transmission and evolution of experimental microcultures in groups of young children. Developmental Psychology, 46(6), 1694-1709. https://doi.org/10.1037/a0020786

Wu, R., \& Kirkham, N. Z. (2010). No two cues are alike: Depth of learning during infancy is dependent on what orients attention. Journal of Experimental Child Psychology, 107(2), 118-136. https://doi. org/10.1016/j.jecp.2010.04.014

Wu, R., Tummeltshammer, K. S., Gliga, T., \& Kirkham, N. Z. (2014). Ostensive signals support learning from novel attention cues during infancy. Frontiers in Psychology, 5, 251-260. https://doi.org/10.3389/ fpsyg.2014.00251

Xu, F., \& Kushnir, T. (2013). Infants are rational constructivist learners. Current Directions in Psychological Science, 22(1), 28-32. https://doi. org/10.1177/0963721412469396

Yoon, J. M., Johnson, M. H., \& Csibra, G. (2008). Communication-induced memory biases in preverbal infants. Proceedings of the National Academy of Sciences of the United States of America, 105(36), 1369013695. https://doi.org/10.1073/pnas.0804388105

Yurovsky, D., \& Frank, M. C. (2017). Beyond naïve cue combination: Salience and social cues in early word learning. Developmental Science, 20(2), e12349. https://doi.org/10.1111/desc.12349

Ziv, M., \& Frye, D. (2004). Children's understanding of teaching: The role of knowledge and belief. Cognitive Development, 19, 457-477. https:// doi.org/10.1016/j.cogdev.2004.09.002

\section{SUPPORTING INFORMATION}

Additional supporting information may be found online in the Supporting Information section.

How to cite this article: Bazhydai M, Silverstein P, Parise E, Westermann G. Two-year-old children preferentially transmit simple actions but not pedagogically demonstrated actions. Dev Sci. 2020;00:e12941. https://doi.org/10.1111/ desc.12941 